Mots. Les langages du politique

$96 \mid 2011$

Les discours politiques. Approches interactionnistes et multimodales

Développement durable, humain. La cohérence discursive des contradictions

Françoise Dufour

\title{
OpenEdition
}

Journals

Édition électronique

URL : https://journals.openedition.org/mots/20284

DOI : $10.4000 /$ mots.20284

ISSN : 1960-6001

Éditeur

ENS Éditions

Édition imprimée

Date de publication : 5 septembre 2011

Pagination : 81-96

ISBN : 978-2-84788-316-9

ISSN : 0243-6450

Référence électronique

Françoise Dufour, "Développement durable, humain. La cohérence discursive des contradictions », Mots. Les langages du politique [En ligne], 96 | 2011, mis en ligne le 05 septembre 2013, consulté le 22 avril 2022. URL : http://journals.openedition.org/mots/20284 ; DOI : https://doi.org/10.4000/mots. 20284 


\section{Développement durable, humain. La cohérence discursive des contradictions}

Dès les années quatre-vingt, en réponse aux nombreuses critiques portées aussi bien dans le champ politique que dans celui de la recherche en sciences sociales (Partant, 1982 ; Latouche, 1991 ; Rist, 1996...), la notion postcoloniale ${ }^{1}$ de développement a vécu différents réaménagements discursifs par le biais d'adjectifs à visée compensatoire (durable, humain, local, équitable, solidaire) et de préfixations (codéveloppement, écodéveloppement).

J'analyserai ici les effets discursifs de deux de ces reformulations - développement durable, développement humain - qui, en raison de leur caractère relativement figé et de leur capacité à «cristallis[er] des enjeux politiques et sociaux», peuvent être qualifiées de «formules» (Krieg-Planque, 2009, p.7). L'analyse portera sur les usages dans le champ des organisations en charge de l'aide au développement au niveau bilatéral ou multilatéral dans des textes à signature institutionnelle (ONU, Parlement européen...) ou signés de représentants d'organismes œuvrant dans ce champ (Boutros Boutros-Ghali, Jacques Chirac, Alain Juppé...).

Ma contribution de linguiste et d'analyste du discours, dans ce champ déjà bien investi par les sciences humaines et sociales, consiste à étudier le fonctionnement discursif des adjectifs à caractère argumentatif qui composent ces formules : durable et humain, par comparaison avec des adjectifs à fonctionnement déterminatif tels que économique, social, commercial...

Je montrerai comment les «opérateurs de neutralisation de la conflictualité » (Krieg-Planque, 2010) que sont les formules «développement durable» et «développement humain» contribuent à la mise en cohérence discursive des contradictions constitutives de l'historicité de la notion de développement, tout en reconduisant le statu quo de l'ordre du monde inscrit dans le noyau notionnel et mis en scène dans l'agencement énonciatif.

1. Postcolonial sans tiret ne réfère pas à une vision historique mais plutôt à une situation politique née dès la fin des années cinquante et qui s'est concrétisée avec la Conférence des pays non alignés, dite Conférence de Bandoeng (1955).

Praxiling (UMR 5267 CNRS et Université Montpellier 3) francoise.dufour@univ-montp3.fr 


\section{Les tribulations linguistiques de la notion de développement}

Je m’intéresserai d'abord au terme développement ainsi qu'aux constructions syntaxiques auxquelles il a donné lieu dans un corpus de textes caractérisé par sa diversité et son épaisseur historique. Le terme trouve en effet son origine 2 dans l'idéal des «progrès de la civilisation» (Condorcet, 1794, p.113) pensé par les Lumières comme une amélioration linéaire de la condition humaine. D’abord repris au service de la nécessité économique et de la raison coloniale, il devient enfin le mot d'ordre international dans la perspective de l'intégration à l'économie mondiale des nouveaux États issus des indépendances et est déclaré comme un «droit inaliénable de l'homme » par le Haut commissariat aux droits de l'homme de l'ONU (1986).

\section{L'ambiguïté de la nominalisation développement. Action et résultat}

Le développement est appréhendé comme une notion. De nature hybride, la notion est composée de représentations expériencielles, sociales, culturelles, interdiscursives (Culioli, 1990, p. 21). En tant que telle, elle n'est pas dicible en soi (Franckel, 1998, p. 57). Les valeurs qui la composent (le progrès économique, la démocratie, la sécurité, le niveau de vie...), elles-mêmes objets de «dissensions multiples» (Foucault, 1969, p. 203), sont capitalisées, en deçà des différentes expressions formulaires, à un niveau "prédiscursif» (Paveau, 2006, p.14). La notion peut s'exprimer par le prédicat : 〈avoir les propriétés d'un être développé〉, ou par le terme qui la représente le mieux ou « terme opérateur» (Achard, 1989, p. 822) : le développement.

Le nom développement est issu d'un processus de nominalisation par adjonction suffixale à un radical verbal. L'étymon desvelopper (fin du $12^{\text {e }}$ siècle) : «ôter de l'enveloppe», donne le processus de développement constitutif de l'espèce humaine, "en puissance», à l'instar des phénomènes biologiques : «Développer : faire s'accomplir ce qui est en puissance » (Académie française, 1992) ; «extension progressive de ce qui a commencé» (Grand Larousse, 1982).

Ce programme de sens du radical verbal autorise à penser la quête d'un infini, comme l'avait relevé Serge Latouche (2004, p. 68).

Par son origine verbale, développement a la capacité de dire un procès en Faire, une action, le processus de développer (ex. 1) et, par son statut nominal, celle de dire un procès en Être, résultat du procès en Faire, un état de développement (ex. 2) :

2. Pour un historique lexicographique et encyclopédique, voir Dufour, 2007b. 
(1) Il ne s'agit rien de moins [...] que d'établir les bases d'un nouveau contrat mutuel de développement pour le $21^{\mathrm{e}}$ siècle. (Juppé, 2003) 3

(2) Cela est vrai non seulement pour les pays qui partent d'un faible niveau de développement humain, mais aussi pour les pays qui ont déjà atteint un certain degré de développement. (PNUD, 1990, p.46)

Dans l'exemple 1, Alain Juppé, en mission au Mali dans le cadre de la nouvelle définition des politiques de développement de la France en lien avec le Nouveau partenariat pour le développement de l'Afrique (NEPAD), représente le développement comme une action déjà en cours reprogrammée dans le futur de l'après-signature d'un contrat. En revanche, dans l'exemple 2, le PNUD rend compte d'états de développement évalués antérieurement au discours.

Cette double potentialité de sens est relevée dans le premier Rapport sur le développement humain :

(3) Ici, l'expression développement a trait au processus 4 d'élargissement des choix des individus et au niveau atteint de bien-être. (PNUD, 1990, p. 11)

\section{Les modificateurs du nom développement et leurs effets de sens}

Afin de montrer les spécificités des adjectifs durable et humain, il est nécessaire de les comparer aux autres formes d'expansion - noms, adjectifs - ainsi qu'à l'usage sans expansion. Le tableau ci-dessous reprend les différentes formes avec des exemples dont l'indexation renvoie aux textes cités infra.

\begin{tabular}{|c|c|}
\hline Types d'expansion & le développement \\
\hline Un complément de nom & $\begin{array}{l}\text { de notre colonie, de nos TOM, des nations pauvres, de } \\
\text { l'Afrique, des pays en voie de développement (ex. } 4 \text { à 8) }\end{array}$ \\
\hline Un adjectif restrictif & $\begin{array}{l}\text { économique, social, industriel, agricole ou commercial } \\
\text { (ex. } 9 \text { à 12) }\end{array}$ \\
\hline Un emploi absolu & le développement ø (ex.13 à 16) \\
\hline Un adjectif explicatif & durable, humain (ex. 17 à 27) \\
\hline
\end{tabular}

Tableau 1. Les différents types d'expansion du terme-notion développement

\section{Développement de $\mathrm{x}$ : la désignation de la référence}

Dans le cas des expansions avec un complément de nom, l'extension est restreinte à des territoires (colonie, TOM, nations pauvres, Afrique, pays en voie de développement) :

3. Tribune publiée dans la presse africaine. Les références précises du corpus figurent en fin d'article.

4. Le PNUD souligne. 
(4) En reliant cette ville [...] au Niger par une voie rapide au bord de l'océan, le développement de notre colonie marchera bientôt à pas de géant. (Bunge 5,1891$)$

(5) [...] le développement de nos TOM [...] (Giscard d'Estaing6 ${ }^{6}, 1950$, p. 447)

(6) Les pays riches estimaient que leur participation à l'effort du développement des nations pauvres [...] (Rullière7, 1958)

(7) Il est certain, en effet, que le développement de l'Afrique ne peut que bénéficier à l'Europe. (Decupper $\left.{ }^{8}, 1970\right)$

(8) [...] le développement des pays en voie de développement. (ONU, 1974)

L'origine verbale de la nominalisation développement permet au complément de nom d'assumer la fonction de sujet aussi bien que celle d'objet du procès de développement. À partir de l'énoncé 7, il est possible de reconstruire deux types d'énoncés sous-jacents :

- 〈le fait que l'Afrique se développe ne peut que bénéficier à l’Europe〉(7a), dans lequel l'Afrique est sujet et agent responsable du procès de développement;

- 〈le fait de développer l'Afrique〉 (7b), dans lequel le nom est objet et patient du procès de développement.

Cette ambiguïté reflète la situation paradoxale dans laquelle se trouvent les deux parties (Nord et Sud) dans les programmes d'aide au développement (Dufour, 2009). Elle constitue une première source de contradiction inhérente à la forme de la nominalisation développement : qui est acteur du développement?

\section{Économique, social... : des épithètes restrictives}

Les adjectifs économique, social, industriel, agricole ou commercial, glosables par des noms, visent à restreindre l'extension à des secteurs du développement :

(9) Ce pays peut produire en abondance les mils ou sorghos, le bétail, l'arachide, l'indigo et même le coton. Mais l'autorité à laquelle il est soumis s'oppose ellemême à tout développement agricole ou commercial. (Chasseloup-Laubat9, 1860)

(10) [...] l'établissement sans retard d'un fonds des Nations unies pour le développement économique. (Conférence de Bandoeng, 1955)

(11) Les dirigeants des pays attardés eux-mêmes [...] allaient être séduits par cette perspective d'un développement industriel sans sacrifice national. (Rullière, 1958)

5. Membre de la Société de géographie commerciale du Havre, Edouard Bunge a défendu l'idée d'une loi dite de «prime coloniale» pour le négoce et l'export de produits d'origine sénégalosoudanienne vers la France.

6. Edmond Giscard d’Estaing (1894-1982), père de l'ancien président de la République, président du Comité français de la Chambre de commerce internationale de 1947 à 1959.

7. Auteur d'un ouvrage consacré à la mise en valeur des pays sous-développés de l'Union française par la modernisation de l'agriculture.

8. Fondateur de la revue Africa (Dakar).

9. Ministre de l'Algérie et des Colonies. 
(12) L'instauration d'un nouvel ordre économique international [...] assurera dans la paix et la justice aux générations présentes et futures un développement économique et social qui ira en s'accélérant. (ONU, 1974)

La détermination économique est largement majoritaire, si l'on considère que les adjectifs agricole, industriel et commercial identifient des sous-domaines du registre-maître de l'économie. En outre, lorsque les deux qualificatifs économique et social sont coordonnés, la systématicité de l'ordre10 indique une relation de consécution entre économique et social.

La fonction des expansions analysées supra est de modifier l'extension du nom développement et par conséquent de fixer un terme ou déterminer le contenu notionnel du nom : des limites sont ainsi données à l'ancrage du référent au monde (Goes, 1999, p. 82).

\section{Le développement ø : l’essentialisation de la notion}

L'usage du nom sans épithète s'impose avec la formule «programme de développement» dans le «discours sur l'état de l'Union» du président des États-Unis, Harry Truman, le 20 janvier 1949. Puis, dans les discours dits « du développement», en particulier dans les discours des théoriciens tels que François Perroux et ceux des organisations internationales :

(13) L'économiste à qui l'on demande : «Qu'est-ce que le développement?» doit à mon sens répondre : Le développement est la combinaison des changements mentaux et sociaux d'une population qui la rendent apte à faire croître, cumulativement et durablement, son produit réel global. (Perroux, 1960)

(14) Programme des Nations unies pour le développement.

(15) Le développement a pour objectif fondamental de créer un environnement qui offre aux populations la possibilité de vivre longtemps, en bonne santé. (PNUD, 1990, p.9)

Cet usage témoigne de l'évidence d'un sens partagé au sein d'une communauté discursive chez qui le terme fait écho. L'absence de caractérisation signe la structuration d'un ensemble de représentations, autrement dit l'existence d'une notion. Son actualisation discursive par la détermination définie le fait état de l'essentialisation de la notion qui se constitue en idéologie ${ }^{11}$ : le propos n'est pas le développement d'un territoire, d'un secteur économique, mais le développement en soi et pour soi.

10. Sur les 34 occurrences relevées, aucune ne se présente dans un ordre inversé - social et économique.

11. Progrès et civilisation ont suivi la même évolution au $19^{\mathrm{e}}$ siècle. 


\section{Durable, humain : des caractérisants argumentatifs}

À partir de la décennie 1980, en réaction, le développement devient alors "durable» ou "soutenable» selon l'option de traduction choisie pour sustainable development ${ }^{12}$ (Brundtland, 1987), et «humain» (PNUD, 1990). Ces expansions adjectivales peuvent sembler identiques à celles qui sont analysées supra. Pourtant, les adjectifs durable et humain assument une autre fonction : celle de soutenir le projet de redéfinition de la notion de développement à partir des référentiels antérieurs. Alors que les épithètes économique, social... pouvaient être glosées par des noms sectoriels, durable et humain sont reformulés par des relatives qui mettent en évidence des propriétés constitutives de la notion mais qui ne vont plus de soi (Leeman, Meleuc, 1984, p. 33). En effet, si le processus de développement est une activité dont les bornes initiales et finales ne sont pas indiquées (rien n'indique que le procès soit censé s'arrêter), les états résultatifs de développement témoignent d'une dégradation de la nature et de l'humain qui sont aptes à compromettre la durabilité du développement, tel qu’il est défini dans la déclaration sur le droit au développement :

(16) Le développement est un processus global, économique, social, culturel et politique, qui vise à améliorer sans cesse le bien-être de l'ensemble de la population et de tous les individus, sur la base de leur participation active et significative au développement et au partage équitable des bienfaits qui en découlent. (ONU, 1986)

Dans les premières années de l'émergence de la caractérisation durable, les locuteurs adjoignent des reformulations explicatives : qui soit durable, c'està-dire qui peut perdurer13 :

(17) [...] obtenir un développement global qui soit durable [...] en guidant le monde sur une voie de développement qui soit durable. (Brundtland, 1987)

(18) La commission mondiale pour l'environnement et le développement a défini le développement durable comme un développement qui répond aux besoins actuels sans limiter l'aptitude à répondre aux besoins des générations futures. (PNUD, 1992, p.19)

(19) Le plaidoyer pour un développement durable, c'est-à-dire qui peut être soutenu dans le temps. (PNUD, 1992, p.18)

Avec le temps, la reformulation explicative devient caduque :

(20) Il convient de faire preuve de prudence dans la gestion de toutes les espèces vivantes et de toutes les ressources naturelles, conformément aux préceptes du développement durable. (ONU, 2000)

12. Pour les motivations des traductions françaises de sustainable, voir Chansou, 1994, p. 101-102.

13. Durable est un adjectif temporel dérivé de la forme verbale durer. 
La formule "développement humain» est introduite en 1990 par le PNUD qui « décide de produire un rapport annuel sur la dimension humaine du développement» (William H. Draper14, PNUD, 1990, p. 3) dénommé «Rapport mondial sur le développement humain ». Alors qu'antérieurement, avec la forme développement $\varnothing$, l'élévation du niveau de vie des populations comme finalité des politiques de développement allait sans dire, avec la formule «développement humain ", la place de l'humain est explicitement réinscrite au centre de la notion de développement. Ce projet se concrétise notamment par l'instauration de l'indicateur de développement humain (IDH), indicateur synthétique (longévité, niveau d'éducation et revenu par habitant) conçu pour mieux rendre compte des effets du progrès que le PNB, qui mesure les seules performances économiques des pays. L'épithète humain a pour fonction de soutenir et d'alimenter la définition notionnelle du développement, au risque de la tautologie :

(21) Le développement humain est un processus qui conduit à l'élargissement de la gamme des possibilités qui s'offrent à chacun. [...] Le développement humain reprend donc à son compte bon nombre des éléments constructifs des approches antérieures. Cette large définition permet de mieux saisir la complexité de la vie de l'homme. (PNUD, 1990, p. 12)

La promotion d'un développement (centré sur l')humain s'inscrit discursivement à travers des modalités déontiques fondées sur une instance morale qui dicte ce qu'il convient de faire. L'obligation du devoir n'est pas assumée énonciativement par des actants humains mais par le développement lui-même :

(22) Des considérations techniques relatives aux moyens de réaliser le développement humain [...] ont parfois dissimulé le fait que le développement doit avant tout profiter aux individus. (PNUD, 1990, p. 9)

(23) Le développement doit donc être bien plus qu'une accumulation de revenus et de richesses. Il doit être centré sur les personnes. (Ibid., p. 10)

L'introduction des caractérisations durable ou/et humain entend pointer la nécessité de (re)placer la nature et l'humain au centre du projet de développement. Humain/durable s'oppose à (exclusivement) économique, comme l'explique le secrétaire général de l'Organisation internationale de la francophonie, Boutros Boutros-Ghali, dans le cadre du Forum des solidarités Nord-Sud:

(24) Le plus important reste, sans doute, l'élargissement du concept de développement. Il est, en effet, désormais reconnu et accepté de tous que le développement est un phénomène global qui ne saurait se réduire à sa seule dimension économique. La croissance économique reste le moteur du développement, mais il faut aussi prendre en compte ces autres dimensions que sont l'environnement, la justice sociale, la démocratie et la paix. (Boutros-Ghali, 2000)

14. William H. Draper était à l'époque administrateur du PNUD. 
Les deux propriétés humain et durable sont associées dès le premier rapport du PNUD (1990) :

(25) Développement humain réussi et durable. (p.46)

(26) Pour qu'un développement humain soit durable. (p.47)

Et dans les rapports qui suivent, les deux épithètes sont postposées sans coordination :

(27) Le développement humain durable. (PNUD, 1992, p. 17)

(28) Il n'y a pas de contradiction entre développement humain et développement durable. Tous deux sont fondés sur l'universalité du droit à la vie. Les modes de développement qui perpétuent les inégalités actuelles ne peuvent ni ne méritent d'être conservés. C'est la raison pour laquelle le développement humain durable est un concept plus global que le développement durable. (PNUD, 1994, p. 20)

L'ordre de succession des deux épithètes laisse penser que c'est le développement humain qui doit « durer» ou être soutenu dans le temps : développement humain durable = développement centré sur l'humain qui puisse durer. Selon les économistes Jean Gadrey et Florence Jany-Catrice, la conjonction des deux aspects durable et humain tente de concilier des objectifs qui ne sont pas perçus pareillement au Nord et au Sud. La question du développement concerne essentiellement le Sud, et les préoccupations environnementales, le Nord :

La conjonction du développement humain (au sens très large du PNUD) et du développement durable est probablement ce qui, aujourd'hui, suggère le mieux les objectifs politiques des comptes de l'avenir. Ce sont eux qui relient le plus fortement les urgences exprimées par les pays du Sud, les attentes écologistes (pour l'instant portées par certaines catégories sociales des pays du Nord) et le retour des «questions sociales» (pauvreté, inégalités, violences) au Nord et au Sud. (Gadrey, Jany-Catrice, 2005, p.7)

\section{La cohérence de la formule contradictoire développement humain, durable}

La nécessité d'un processus de développement sans fin paraît contradictoire avec les intentions de durabilité et d'humanité énoncées dans les formules développement durable, humain. Cette contradiction n'est pas fortuite.

\section{Un développement [économique] durable, humain}

Les locuteurs qui choisissent de requalifier le développement affichent la volonté d'en contrôler la dérive strictement économique et ses dégâts collatéraux. Cependant, dès le rapport Brundtland qui introduit la formule, la néces- 
sité d'une "soutenabilité » du développement concerne la durabilité des écosystèmes pour autant qu'elle constitue une des conditions de la durabilité du développement lui-même :

(29) Dans son esprit même, le développement durable est un processus de changement par lequel l'exploitation des ressources, l'orientation des investissements, les changements techniques et institutionnels se trouvent en harmonie et renforcent le potentiel actuel et futur des besoins des hommes. (Brundtland, 1987)

Ces analyses valent pour la production du sens de la formule « développement humain ». L'IDH comme alternative au PIB est le porte-drapeau d'un discours qui soutient que le niveau de développement humain d'un pays n'est pas automatiquement corrélé au revenu par habitant :

(30) II n'y a pas de corrélation automatique entre le revenu par habitant d'un pays et son niveau de développement humain. (PNUD, 1990)

(31) Les individus ne sauraient être réduits à leur seule dimension d'homo economicus. (Draper in PNUD, 1990, p. 3)

Pourtant, si la croissance économique n'est plus une fin en soi, elle est mentionnée comme le moyen principal pour atteindre l'objectif de développement humain :

(32) Son message central [du Rapport 1990] est le suivant : si la croissance du Produit National Brut (PNB) est indispensable pour atteindre tous les objectifs humains essentiels, l'important c'est d'analyser comment cette croissance se traduit - ou ne se traduit pas - en développement humain dans différentes sociétés. (Draper, PNUD, 1990, p.3)

(33) La croissance économique, facteur important du développement humain, ne peut favoriser celui-ci que si l'accroissement des revenus privés est équitablement réparti et si la croissance génère une augmentation des recettes publiques investies dans le développement humain. (PNUD, 1999)

L'argumentaire d'une conciliation entre des objectifs a priori contradictoires - la croissance économique, le bien-être de l'humain et la durabilité des milieux naturels - apparaît dans la fréquence des formes concessives relevée par Alice Krieg-Planque dans les constructions discursives actualisant la formule « développement durable», comme dans le rapport Brundtland :

(34) Un mode de développement qui répond aux besoins du présent sans compromettre les capacités des générations futures à répondre aux leurs. (Brundtland, 1986)

Ou encore, dans le discours de Boutros-Ghali (ex. 24 supra), dans la proposition introduite par le connecteur mais qui marque une orientation argumentative à valeur concessive : 
La croissance économique reste le moteur du développement, mais il faut aussi prendre en compte ces autres dimensions que sont l'environnement, la justice sociale, la démocratie et la paix. [Je souligne]

Ces constructions ont pour caractéristique d'exprimer "une contradiction dépassable» (Krieg-Planque, 2010).

\section{Le statu quo dans l'ordre du discours}

Avec le recul, la géographe Sylvie Brunel établit le constat que le développement durable tend à se réduire à sa seule composante environnementale (la protection de la nature) et a pu être envisagé par certains acteurs comme un nouveau marché qui participe à augmenter la richesse des pays riches en excluant un peu plus les pays pauvres :

Une nouvelle guerre froide qui ne dit pas son nom. Elle oppose les riches, les « développés ", ceux qui disposent à la fois du savoir et des moyens, et les pauvres qui en sont exclus. Non seulement le développement durable discrimine [...] mais il stigmatise : les pauvres sont accusés de mal agir. Un nouvel apartheid oppose riches et pauvres, autant géographique que social. (Brunel, 2008, p.11-12)

Ce constat est attesté par les acteurs institutionnels du développement euxmêmes qui déclarent que malgré des «avancées manifestes dans certains domaines », "les interdépendances croissantes entre les nations et les peuples rendent aussi les différences plus criantes» (PNUD, 2002, p.13).

En effet, si «les consensus en matière de représentation économique implicite (sous-jacente aux indicateurs économiques) évoluent» (CAS, 2008, p.4), la promesse de développement durable et humain ne produit pas d'écho en chaîne dans les actes de nomination et dans la distribution des places des acteurs du développement, qui restent rigoureusement stables au fil des rapports du PNUD de 1990 à 2007/2008 :

(35) pays $X$ riches vs pays $Y$ pauvres:

a) Les pays riches et les institutions économiques qu'ils contrôlent [...] font l'impasse sur les autres défis que doivent relever nombre de pays les plus pauvres (PNUD, 2003, p.17).

b) Lutter contre la pauvreté dans le monde n'est pas seulement une obligation morale, cela contribuera aussi à édifier un mode plus stable, plus pacifique, plus prospère et plus juste, témoin de l'interdépendance entre les pays riches et les pays pauvres. (Parlement européen, 2006, p.1)

(36) X riches vs $Y$ en développement:

Les pays riches sont déjà en train de mettre en place des systèmes de santé publique [...]. Cependant, les impacts sur la santé seront ressentis plus profondément dans les pays en développement à cause des taux de pauvreté. (PNUD, 20072008, p.19) 
(37) X industrialisés vs Y en (ou en voie de) développement:

a) Au fur et à mesure de l'avance de nos travaux, le nationalisme et les divisions artificielles entre pays «industrialisés » et «en voie de développement», entre l'Est et l'Ouest, disparurent peu à peu. (Brundtland, 1987, p.4)

b) Les pays en développement ont besoin d'accélérer leur croissance économique [...]; les pays industrialisés, de leur côté, souhaitent poursuivre leur croissance. (PNUD, 1992, p. 20)

(38) X industrialisés vs Y les moins avancés:

Nous nous engageons également à prendre en compte les besoins particuliers des pays les moins avancés. [...] Nous demandons aux pays industrialisés [...] (ONU, 2000)

(39) X riches vs X' nouvellement industrialisés vs Y les plus pauvres:

La majorité des habitants des pays les plus pauvres se préoccupent de survivre. Les habitants des pays nouvellement industrialisés s'efforcent d'acquérir des qualifications supplémentaires [...]. Les populations des pays riches songent peut-être davantage aux questions sociales. (PNUD, 1992, p.14)

L'échantillon d'énoncés supra reflète l'organisation énonciative typique des discours du développement depuis les années soixante et les indépendances. Les dénominations des catégories d'acteurs, qui ne sont pas que des mots, mais «des traces d'acte de rapport social» (Achard, 1989, p. 800), donnent à voir le spectacle linguistique d'un ordre du monde qui s'organise comme représenté dans le tableau 2.

\begin{tabular}{ll} 
Position haute (types) & Position basse \\
\hline riches & pauvres, les plus pauvres \\
industrialisés & moins ou les moins avancés \\
développés & en (ou en voie de) développement \\
\hline
\end{tabular}

Tableau 2. Paradigmes dénominatifs des différentes catégories d'acteurs

Les acteurs en position haute (pays riches, industrialisés, développés) sont agents de procès en Faire d'action dominante : $\langle X$ agir $\rangle$ ou $\langle X$ agir sur $Y\rangle$ : bloquer $Y$, exercer des pressions sur $Y$, contrôle; aider $Y$; annoncer, appeler à.

Les acteurs en position basse (pays pauvres, en développement, les moins avancés) sont patients de procès en Être dominé :

- 〈Y être〉: être confrontés à ;

- 〈Y verbe de dépendance〉 : avoir besoin, réclamer une aide supplémentaire à ;

- ou de procès négatifs du type 〈Y ne pas agir $\rangle$ : ne pas faire assez d'efforts; ne pas pouvoir.

L'opposition récurrente des caractérisants riches et industrialisés à ceux de pauvres ou en développement rend compte de la prégnance de la valeur économique sur tout autre déterminant. Le choix de la désignation nouvellement 
industrialisés (ex. 39) vient argumenter en cette faveur : la production de cette catégorie dite «émergente » est déterminée par la propriété 〈être industrialisé> qui relève de la sphère économique.

Dans l'organisation énonciative, les représentations du «monde développé» - pays riches, industrialisés - occupent la place de «types» (Culioli, 1999, p. 12) ou meilleurs exemplaires de la catégorie X développés, représentatifs de la valeur par excellence, celle d'un idéal notionnel de développement durable et humain. Les modes de dénomination les constituent comme des parangons pour les pays en position basse. Ce sont les types qui véhiculent la valeur rectrice des discours : la richesse et l'industrialisation.

\section{La cohérence des contradictions comme principe d'historicité du discours}

Les différents constats qui sont établis à partir de 2002 mettent à mal les objectifs assignés par la Commission mondiale sur l'environnement et le développement : « un programme global de changement» (Brundtland, 1987). L'ordre du monde semble en contradiction avec l'éthique du vivant (de la nature, de l'humain) prônée dans les déclarations d'intention:

(40) [...] préserver et mettre en valeur les ressources de la planète; favoriser un état d'harmonie entre les êtres humains et entre l'homme et la nature. (Brundtland, 1987)

(41) Le concept de développement humain place au centre la personne humaine et traite de l'élargissement des choix accessibles aux individus dans l'optique d'une vie conforme à leurs attentes. (PNUD, 2002)

(42) Le développement doit avant tout profiter aux individus [...] en mettant les gens au centre du processus du développement. (PNUD, 2006)

Malgré l'argumentation portée par les "habits neufs du développement» (Latouche, 2003) - durable, humain -, les modes de nommer, la distribution énonciative des actants et les types de procès qui constituent des formes d'interpellation à valeur d'acte performatif (Achard, 1995, p. 84) montrent la perpétuation d'une forme de «dominance discursive» induite par la surdétermination économique.

L'adjonction de l'adjectif équitable dans la formule développement humain durable et équitable, qui souligne le fait que l'équité ne va pas de soi, vient alimenter le constat d'une insuffisance du changement, voire de la faillite du projet de réorientation du développement vers des valeurs de respect de l'humain et de la nature. Le Rapport 1998 du PNUD l'explique par les carences des postures politiques qui continuent à privilégier un développement essentiellement économique envisagé dans ses résultats financiers :

(43) Cependant, les choix politiques restent dominés par le souci de la croissance économique comme fin en soi. Dans ce contexte, on mesure souvent le succès et 
l'échec à l'aune de la progression du PIB et des résultats des marchés financiers, au lieu d'envisager comment la croissance économique peut contribuer à un développement humain durable et équitable. (PNUD, 1998, p.18)

La raison du durable et de l'humain se trouve en butte à la contrainte d'un processus de développement sans fin adossé à la nécessité économique surdéterminant les autres valeurs. Consécutivement, les pratiques discursives et notamment l'organisation énonciative rendent compte d'un statu quo dans les équilibres des rapports de pouvoir et elles contribuent à le perpétuer.

La question de la faisabilité d'un réaménagement effectif de la notion de développement reste posée. En effet, les contradictions mises en évidence par les formules développement durable, humain, entre une rhétorique humaniste et la nécessité économique qui détermine des places dans l'ordre du discours et dans l'ordre du monde, n'est pas un cas d'espèce. Elle est ancrée dans l'historicité des pratiques sociales et discursives des relations Nord/Sud. Depuis son émergence dans les discours des Lumières, les discours du progrès de la civilisation, puis ceux du développement se sont construits sur cet «entrecroisement de valeurs qu'on ne peut concilier» (Foucault, 1969, p. 197) qui lui est constitutive. Dans le contexte français, elle a trouvé sa première expression dès 1791 et la création de la République et en particulier, dans les débats anti-esclavagistes qui parviennent à faire tenir ensemble abolition de l'esclavage et colonisation (Dufour, 2010, p.108 et suiv.). Puis, au 19 siècle, le processus d'industrialisation contraint l'Europe à chercher des marchés hors de ses frontières (des matières premières et des consommateurs) et à soutenir la nécessaire exploitation des colonies et des colonisés (sujets «attachés » à la République), et dans le même temps, l'argument de l'assimilation républicaine (annonce de leur future accession au statut de citoyens). Avec l'universalisation du projet de développement (entrée en scène des États-Unis, création de l'ONU), la valeur économique continue à jouer un rôle déterminant, comme le montrent les premières résolutions de l'ONU, «Développement économique des pays insuffisamment développés» (198-III) et «Assistance technique en vue du développement économique» (200-III).

Les adjectifs durable, humain manifestent la prise de conscience d'un déficit dans l'orientation prise par la notion de développement, déficit qui menace la raison d'être du développement : «l'extension progressive de ce qui a commencé ». Les caractérisants durable, humain n'ont pas pour fonction de fixer un terme au référent comme le faisaient les déterminations antérieures, mais d'argumenter un recentrage sur des valeurs éthiques qui sont aux fondements de la notion de développement comprise comme les progrès de la civilisation. Pourtant, par ailleurs, le nom développement apporte par sa racine verbale l'idée d'un processus sans borne finale dont la valeur économique est devenue le moteur essentiel, même si le déterminant économique a été effacé. En 
conséquence, la contradiction que laisse apparaître l'appariement du projet de développement avec des arguments de durable, humain, équitable est rendue cohérente dans sa mise en perspective avec l'historicité discursive de la notion qui se construit et avance en appui sur ces valeurs contradictoires et néanmoins constitutives :

[La contradiction] se révèle finalement comme principe organisateur : c'est pour la fuir, alors qu'elle renaît sans cesse à travers lui [le discours], qu'il se poursuit, et qu'il recommence indéfiniment; c'est parce qu'elle est toujours en deçà de lui, et qu'il ne peut donc jamais la contourner entièrement, qu'il change, qu'il se métamorphose, qu'il échappe de lui-même à sa propre continuité. La contradiction fonctionne alors, au fil du discours, comme le principe de son historicité. (Foucault, 1969, p. 197)

\section{Références}

\section{Bibliographie}

ACHARD Pierre, 1989, «La passion du développement. Une analyse de l'économie politique », thèse de lettres sous la direction de Paul-Henri Chombart de Lauwe, Université Paris 7.

- 1995, «Formation discursive, dialogisme et sociologie », Langages, nº 117, p. 83-95.

BRUNELSylvie, 2008, À qui profite le développement durable?, Paris, Larousse.

Culıoli Antoine, 1990, Pour une linguistique de l'énonciation, t. I, Opérations et représentations, Gap, Ophrys.

- 1999, Pour une linguistique de l'énonciation, t. III, Domaine notionnel, Gap, Ophrys.

DUFOUR Françoise, 2007a, "Dire le "Sud". Quand nommer l'autre catégorise le monde », Autrepart, n० 41, p. 27-39.

— 2007b, «Des rhétoriques coloniales à celles du développement. Archéologie discursive d'une dominance», thèse de sciences du langage sous la direction de Paul Siblot, Université Montpellier 3.

- 2009, «De l'effet de l'interdiscours colonial dans la production du sens de la nomination "pays dits les moins avancés" ", Le sens c'est de la dynamique! La production de sens en psychologie et en sciences du langage, Montpellier, Publications de l'Université Paul Valéry, p. 61-73.

- 2010, De l'idéologie coloniale à celle du développement. Une analyse du discours France-Afrique, Paris, L'Harmattan.

Fou CAult Michel, 1969, L'archéologie du savoir, Paris, Gallimard.

- 1971, L'ordre du discours, Paris, Minuit.

FranCKEL Jean-Jacques, 1998, "Aspects de la théorie d'Antoine Culioli», Langages, n'0 129, p. 52-63.

GADREY Jean, JANY-CATRICE Florence, 2005, Les nouveaux indicateurs de richesse, Paris, La Découverte.

GoEs Jan, 1999, L'adjectif. Entre nom et verbe, Paris, Bruxelles, Duculot. 
KRIEg-PlAnque Alice, 2009, La notion de «formule » en analyse du discours, Besançon, Presses universitaires de Franche-Comté.

- 2010, «La formule “développement durable” : un opérateur de neutralisation de la conflictualité», Langage et société, n0134, décembre, p.5-19.

LATOuChe Serge, 1991, La planète des naufragés. Essai sur l'après-développement, Paris, La Découverte.

- 2003, "L'imposture du développement durable ou les habits neufs du développement ", Mondes en développement, $\mathrm{n}^{0}{ }^{121}$, p. 23-30.

- 2004, Survivre au développement, Paris, Mille et une nuits.

LAZARD Gilbert, 1994, L'actance, Paris, PUF.

LeEmAn Danièle, Meleuc Serge, 1984, "Verbes en table et verbes en -able», Langue française, nº 87, p. 30-51.

PARTANT François, 1982, La fin du développement. Naissance d'une alternative?, Paris, Maspero.

Paveau Marie-Anne, 2006, Les prédiscours. Sens, mémoire, cognition, Paris, Presses de la Sorbonne nouvelle.

RIST Gilbert, 1996, Le développement. Histoire d'une croyance occidentale, Paris, Presses de Sciences Po.

\section{Corpus}

Boutros-Ghal Boutros, 2000, «Une nouvelle ère pour le développement», Aventure, no 90 , automne, 2005.

BRUndtLAnd Gro Harlem, Commission mondiale sur l'environnement et le développement, 1987, Notre avenir à tous, Montréal, Fleuve, 1989.

BUNGE Edouard, 1891, Lettre au sous-secrétaire d'État aux colonies, Eugène Étienne, 31 août (archive manuscrite du Centre des archives d'outre-mer).

CAS (Centre d'analyse stratégique), 2008, «Au-delà du PIB, le bonheur?», La note de veille, nº 91 , février, p. 1-7.

Chansou Michel, 1994, "Développement durable, un nouveau terme clé dans les discours politiques», Mots. Les langages du politique, n³9, juin, Environnement, écologie, Verts, p. 99-105.

Chasseloup-laubat Prosper (de), 1860, Rapport à l'empereur, juin (archive manuscrite du Centre des archives d'outre-mer).

ChIRAC Jacques, 2001, Discours de M. Jacques Chirac, Président de la République, devant la IIle Conférence des Nations Unies sur les pays les moins avancés (Bruxelles), Parlement européen, 14 mai.

CNUCED, 1999, "La communauté internationale entame des négociations sur la prochaine conférence des Nations Unies sur le développement», Communiqué de presse TAD/INF/PR/9932, 25 octobre.

CONDORCET, 1794, Esquisse d'un tableau historique des progrès de l'esprit humain, Paris, GF Flammarion, 1988.

Conférence de Bandoeng, 1955, Communiquéfinal.

DECUPPER Joël, 1970, «Imperfections et carences du FED », Africa, nº 52, p. 35-53. 
Giscard d'Estaing Edmond, 1960 [1950], « Marchés tropicaux il y a dix ans », Marchés tropicaux, n⿳0744, p. 447.

JUPPÉ Alain, 2003, «Vers un nouveau partenariat», mai [http://discours.vie-publique. $\mathrm{fr} /$ notices/033002607.html]. Site consulté le 6 octobre 2010.

ONU, 1970, "Identification des pays en voie de développement les moins avancés», Résolution 2724 (XXV) adoptée sur les rapports de la deuxième commission, 15 décembre.

- 1974, «Déclaration concernant l'instauration d'un nouvel ordre économique international», Résolution 3201 (S-VI).

- 1986, "Déclaration sur le droit au développement», Haut Commissariat aux droits de l'homme, Résolution 41/128, 4 décembre.

— 2008, «Afrique : Assemblée générale. Les pays riches appelés à respecter leurs engagements pour l'Afrique », New York, 22 septembre.

- 2000, Déclaration du Millénaire 55/2, 8 septembre.

Parlement européen (Conseil du Parlement européen et de la Commission sur la politique de développement de l'Union européenne), 2006, «Le consensus européen pour le développement», Journal officiel de l'Union européenne, 24 février.

Perroux François, 1960, «Qu'est-ce que le développement? », Études, n³08, p. 16-33.

PNUD, 1990, «Définir et mesurer le développement humain », Rapport mondial sur le développement humain (ci-après : $R M D H$ ).

- 1992, "Pour une vision nouvelle du développement humain au niveau mondial», RMDH.

— 1994, "Vers un développement humain durable », RMDH.

— 1998, «Où en est le développement humain?», $R M D H$.

— 1999, "Quelle place pour la mondialisation de demain?», RMDH.

— 2000, «Un développement humain axé sur les droits», $R M D H$.

— 2002, "Situation et progrès du développement humain », $R M D H$.

— 2003, «Le pacte du Millénaire pour le développement», $R M D H$.

— 2003, "Les objectifs du Millénaire pour le développement», $R M D H$.

— 2006, «Promouvoir le développement humain», RMDH.

- 2007-2008, «La lutte contre le changement climatique : un impératif de solidarité humaine dans un monde divisé », RMDH.

RULLIÈRE Gilbert, 1958, «La lutte contre le sous-développement, facteur de solidarités économiques nouvelles", Notes documentaires du secrétariat social d'outre-mer, Paris, p.1-16. 